\title{
SENSITIVITY ANALYSIS FOR THE NUMERICAL MODEL OF THE "ROCK THERMOMECHANICS" EXPERIMENT UNDER URF CONDITIONS
}

\author{
Gorelov M. M., Drobyshevsky N. I., Moiseenko E. V., Saveleva E. A., Svitelman V. S. \\ Nuclear Safety Institute of the Russian Academy of Sciences, Moscow, Russia
}

Article received on October 21, 2019

\begin{abstract}
The paper deals with the sensitivity analysis performed for a thermomechanical experiment planned to be carried out at the URF in the Nizhnekanskiy massif. Results of the sensitivity analysis are intended to be applied in the development of an experimental unit. The numerical thermomechanical model is implemented in FENIA numerical code, the sensitivity analysis is performed by means MOUSE code.
\end{abstract}

Keywords: radioactive waste, thermomechanical experiment, numerical simulation, sensitivity analysis, Underground Research Facility (URF), RW disposal.

\section{Introduction}

Calculation and prognostic modeling is seen as a main tool used to develop arguments regarding the long-term safety of RW disposal facilities. Results of studies performed in repository siting area, as well as laboratory studies and field experiments are used to substantiate parameter values of models describing the properties of disposal system elements (geosphere, engineered barriers, radioactive waste, etc.) and the processes occurring in them.

A complex research program has been developed for a deep RW disposal facility (DDF RW) planned for construction in the Nizhnekansk granite-gneiss rock mass (Krasnoyarsk Territory) involving over 150 research tasks with each of them being associated with a specific aspect discussed in the longterm safety case [1]. A significant part of these efforts is going to be implemented in the underground research facility (URF). This R\&D program should provide data required to address all tasks of computational and prognostic modeling enabling a fully-fledged safety assessment and safety demonstration for the proposed facility.

Designs of experimental installations required for the planned URF field studies are currently being developed. Numerical models describing their operation may be helpful in elaborating the designs of proposed experiments, namely: number, characteristics and location of sensors inside the engineered barriers and the rocks, heat release parameters of RW simulators, modes for sensor readout, etc.

This paper focuses on the sensitivity of a model describing possible layout of a thermomechanical experiment planned in the URF to study the impacts produced by thermal and mechanical stresses on the rocks. Numerical model of the experiment is implemented in FENIA code (Finite Element Nonlinear Incremental Analysis) [2] and the sensitivity analysis is performed using MOUSE code (MOdeling Uncertainty and SEnsitivity) [3]. The paper aims to assess the sensitivity of the numerical model to 
variations in rock characteristics and parameters of the installation considered as potentially important for the experiment. Resulting estimates will be applied during R\&D planning.

\section{Description of the Rock \\ Thermomechanics experiment}

Rock Thermomechanics experiment is focused on thermal and mechanical properties of the host rock. The experiment will reproduce thermal effects caused by energy release occurring due to the radioactive decay of substances contained in the waste.

The experiment was proposed to be implemented in a horizontal URF tunnel excavated at the target horizon level of the planned DDF RW. The tunnel arch is supposed to be cylindrical; its width would account for $6 \mathrm{~m}$ with its height ranging from 6 to $6.5 \mathrm{~m}$.

In the center of the tunnel, two vertical boreholes with a diameter of $1.3 \mathrm{~m}$ will be drilled to a depth of $5 \mathrm{~m}$ in the tunnel floor spaced by $4 \mathrm{~m}$ from each other. Each borehole will be fitted with a $2-6 \mathrm{~kW}$ electric heater - a simulator of a heat emitting RW package (Figure 1, left). Sensors recording changes in temperature, pressure, stress and rock movements during the experiment will be fitted within the rocks surrounding the boreholes.

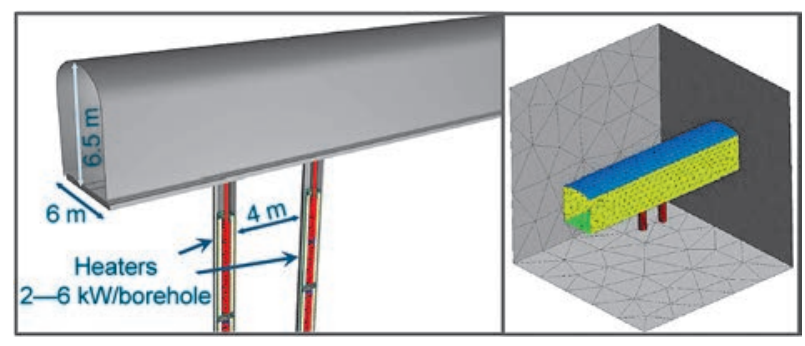

Figure 1. Layout of the Rock Thermomechanics experiment: experimental installation (left) and computational domain (right)

The experiment will be implemented as follows:

1) container fitted with a heating element will be installed into the first borehole and the rock mass will start being heated. At this stage, with only one heater being enabled, the temperature and stress fields will have a fairly simple form, and based on the measurements, a numerical model simulating temperature and stress field evolution will be calibrated taking into account the thermomechanical properties of the rock mass;

2) the heater fitted within the second borehole will be turned on in a year allowing to simulate alternate waste package emplacement into DDF RW and corresponding temperature and stress field evolution;
3) the rock mass will be heated uniformly by two heaters until a quasi-stationary state is reached (presumably for $2-3$ years);

4) at the final stage heating elements will be disabled and the bedrock will be cooling down under natural conditions.

\section{Numerical model of the thermomechanical experiment}

Numerical simulation of the experiment is implemented in a finite element code FENIA 3D providing concurrent simulation of thermomechanical processes.

Two problems are to be solved under concurrent simulation of thermal and mechanical impacts on rocks: time-dependent changes in the temperature and stress fields are to be calculated. Moreover, temperature changes affect the stress-strain state and this effect is associated with thermal expansion and the interdependences between the mechanical properties of materials and the temperature. The opposite effect - stress-strain state changes along the temperature field - is considered to be negligible. Under such assumptions, a flow chart can be developed to solve these problems: for each time step, heat distribution is calculated first, followed by stress calculations accounting for previously calculated temperatures.

Problems associated with heat transfer modeling and stress strain evaluations are solved based on an equivalent variational formulation using finite element approach $[4,5]$ suggesting that the modeled domain is split into finite elements. Under such a representation, the temperature field is described by nodal values and the stress and strain fields are represented by symmetric tensors.

In general case, energy conservation equation is used to calculate the temperature field:

$$
\begin{gathered}
\rho(t, X) c(t, T, X) \frac{\partial T}{\partial t}-\frac{\partial}{\partial x_{i}}\left(\lambda_{i j}(t, T, X) \frac{\partial T}{\partial x_{j}}\right)=q(t, X), \\
i, j=1 \ldots 3
\end{gathered}
$$

where $T$ is temperature $[\mathrm{K}], \rho$ is density $\left[\mathrm{kg} / \mathrm{m}^{3}\right]$, $c$ is specific heat capacity $[\mathrm{J} /(\mathrm{kg} \cdot \mathrm{K})], \lambda$ is thermal conductivity tensor $[\mathrm{W} /(\mathrm{m} \cdot \mathrm{K})], q$ is heat flux at the boundary. Under numerical simulation of the rock thermomechanics experiment, equation (1) is applied in its simplified form, properties of individual materials are assumed as uniform in space not being directly dependent on time.

Relations between stress tensor $\sigma$ and strain tensor $\varepsilon$ account for the dependences between model coefficients and temperature and thermal strains. 
Thermal strain increment $\mathrm{d} \varepsilon^{T}$ is expressed through volumetric thermal expansion coefficient $\alpha$, which is also a temperature-dependent parameter:

$$
\mathrm{d} \varepsilon^{T}=\left[\frac{\mathrm{d} \alpha}{\mathrm{d} T}\left(T-T_{0}\right)+\alpha\right] \mathrm{d} T .
$$

In the FENIA code, depending on the considered material, relationship between stress and strain tensors can be specified by a number of models: elastic, elastic-plastic and visco-plastic. To simulate the considered experiment, applied was an elastic material model.

Under numerical simulation of the rock thermomechanics experiment, a large section of rocks was assumed as a computational domain so that its size would be large enough to be able to accept zero heat fluxes at its boundaries. A grid adjusted to the experiment geometry was plotted in the computational domain (Figure 1, right) - being sparse near the boundaries of the domain and sophisticated inside the experimental zone. To account for the effect produced by ventilation, boundary condition of the third kind was set for tunnel surfaces (floor, walls and roof). Temperature of the cooling air was assumed as constant being equal to $9^{\circ} \mathrm{C}$; heat transfer coefficient varied under the sensitivity analysis.

Modeling was performed only for the first three stages of the experiment. Cooling process was not simulated. Thus, the following stages of rock state evolution were simulated: rock heating by one heater; enabling of the second heater and associated changes in the temperature and stress fields and attainment of a quasi-stationary state.

\section{Assessment of thermomechanical experiment model sensitivity to its parameters}

Numerical model sensitivity to its parameters can be evaluated via the degree of their influence on the simulation result uncertainty. This paper suggests the use of a variational approach to the sensitivity assessment. It assumes that the uncertainty is characterized by relevant variation in the output result. Whereas, the contribution of the input parameter to this variation affects the degree of model sensitivity to its changes.

Direct contribution of the parameter to the output variation is estimated using the first order index [6]:

$$
S_{i}=\frac{V_{X_{i}}\left[E_{X_{\sim i}}\left\{y \mid x_{i}\right\}\right]}{V(y)}=\frac{V_{i}}{V(y)},
$$

where $E_{X_{\sim}}\left\{y \mid x_{i}\right\}$ is an averaged simulation result when all parameters are considered as varying except for $x_{i} ; V_{X_{i}}$ stands for the variation in different values of parameter $x_{i} ; V(y)$ is an unconditional variation of the model output.

In a generic form, complete output variation can be represented as a decomposition:

$$
V=\sum_{i} V_{i}+\sum_{i<j} V_{i j}+\sum_{i<j<m} V_{i j m}+\cdots+V_{123 \ldots M}
$$

where $V_{i j}=V\left(E\left\{y \mid X_{i}, X_{j}\right\}\right), V_{i j m}=V\left(E\left\{y \mid X_{i}, X_{j}, X_{m}\right\}\right)$ and etc. are indices of intermediate orders (second, third, etc.) specifying joint contribution of parameter groups to the output variation.

If the model is additive, expression (4) can be reduced to:

$$
V=\sum_{i} V_{i} \text { or } \sum_{i} S_{i}=1 .
$$

If the model is non-additive, i.e. condition (5) is not satisfied, then the degree of parameter influence can be higher due to the effects produced on calculation result variation together with another parameter (or group of parameters).

Unconditional identification of parameters with negligible contribution to the result variations can be provided through the use of indices specifying total contribution of the input parameter depending not only on its direct contribution, but also on the effects produced by its interaction with all other parameters:

$$
S_{i}^{T}=\frac{E_{x_{\sim i}}\left[V_{x_{i}}\left(y \mid x_{\sim i}\right)\right]}{V(y)}=\frac{V_{T_{i}}}{V(y)} .
$$

Zero value of the total contribution index is seen as a necessary and sufficient condition demonstrating that the parameter does not produce any influence on the simulation result. A value below 0.01 allows to state the negligibility of the parameter.

In this paper, variational approach to sensitivity analysis was implemented using the Sobol method [6]. Computational complexity is seen as a main disadvantage of the method: more model runs are required to ensure adequate index estimates.

Four time points were assumed under the sensitivity analysis: 60, 300, 400 and 1,000 days. These milestones correspond to an unsteady state after the first heater was enabled, steady field of temperatures and stresses that evolved under single heater operation, unsteady state after the second heater was enabled and quasi-stationary state of the rock when both heaters were on. Simulation results were explored at the three points of the computational domain (Figure 2):

1) in-between the boreholes at a depth of $2.5 \mathrm{~m}$, corresponding to one half of the borehole's depth; 


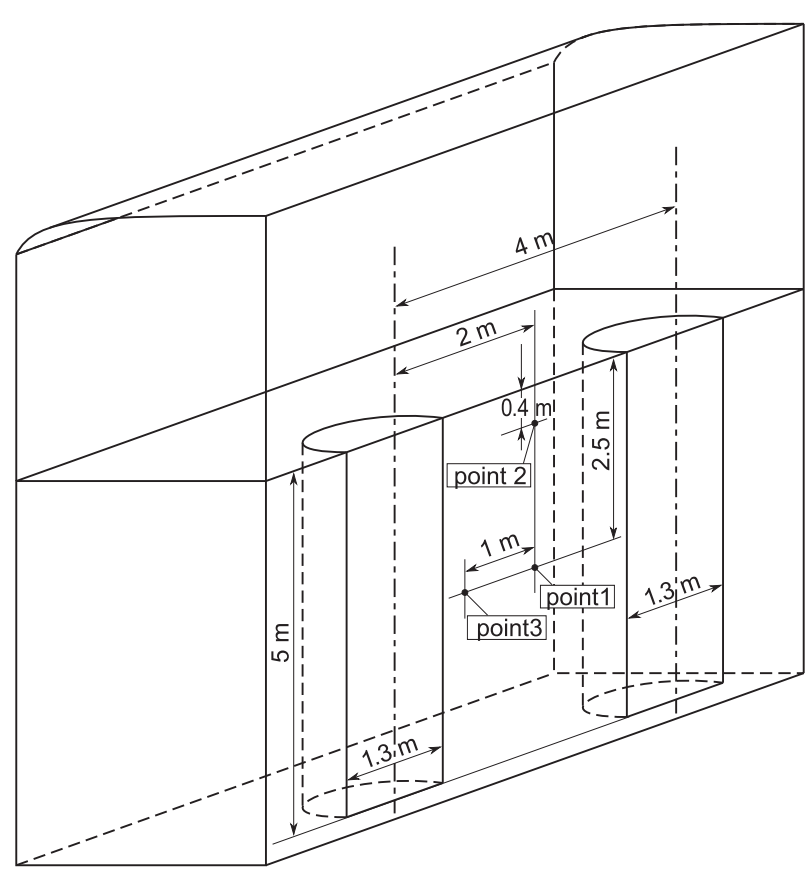

Figure 2. Layout of points set to assess the sensitivity of calculation results to the input parameters

2) above the first one at a depth of $0.4 \mathrm{~m}$ from the tunnel floor;

3) at a distance of $1 \mathrm{~m}$ from the first point towards the borehole fitted with the heater that was enabled first.

Numerical model sensitivity was estimated to such input parameters as heat output from heaters, heat conductivity and heat capacity of rocks, heat transfer coefficients from the floor, walls and roof surfaces, rock thermal expansion coefficient and corresponding Young's modulus. Table 1 presents ranges of parameter variations. For sensitivity assessment purposes, a total of 16 thousand computational model runs was implemented.

Table 1. Variable parameters under the sensitivity assessment

\begin{tabular}{|l|c|c|}
\multicolumn{1}{|c|}{ Parameter } & Minimum & Maximum \\
\hline Heat output, $\mathrm{W} / \mathrm{m}^{2}$ & 301.4 & $1,356.1$ \\
\hline Thermal conductivity, $\mathrm{W} /(\mathrm{m} \cdot \mathrm{K})$ & 2.33 & 3.49 \\
\hline Heat capacity, $\mathrm{J} /(\mathrm{kg} \cdot \mathrm{K})$ & 672 & 1,008 \\
\hline $\begin{array}{l}\text { Heat removal from the surface of tunnel } \\
\text { floor, walls and roof, } \mathrm{W} /\left(\mathrm{m}^{2} \cdot \mathrm{K}\right)\end{array}$ & 1 & 50 \\
\hline $\begin{array}{l}\text { Linear coefficient of thermal expansion, } 1 / \mathrm{K} \\
\text { Young's modulus, } \mathrm{Pa}\end{array}$ & $8.0 \mathrm{e}-5$ & $1.2 \mathrm{e}-4$ \\
\hline
\end{tabular}

Figures 3-6 present the sensitivity assessment results.

Figures 3 and 5 present the breakdown of firstorder sensitivity indices illustrating the contribution of parameter variation to the model result variation (temperature and effective stress, respectively). Each line features images related to one of the spatial points considering different calculation times. Figures 4 and 6 show the bar charts presenting first-order indices and full contribution of the parameters under temperature and thermal stress calculations, respectively. As can be seen from the pie charts, the models applied to calculate temperatures and stresses are non-additive: white section in the diagram indicates the interaction effect. At the same time, bar graphs (Figures 4 and 6) demonstrate that the interaction effect does not affect the index ratios and is only manifested by the combined influence of the same parameters.

As can be seen from the sensitivity assessment of temperature calculation model (Figures 3 and 4), heat release from the heating element appears to be a most important parameter for all three points considered and for the four milestones discussed above. The role of thermal conductivity at the first and second points (located at an equal distance from the heaters) increases at later times, and at the third point (next to the first heater), thermal conductivity effect is approximately the same for all the time points considered. This is explained by the fact that at the first point the temperature sets up quickly and later suffers only some minor changes. Parameter influence describing heat removal from the tunnel floor, walls and roof turned out to be significant only for the second point. Such an effect produced by heat removal can be explained by its location (at a depth of $0.4 \mathrm{~m}$ from the tunnel floor) with the rest of the points being located at a depth of about $2.5 \mathrm{~m}$. Any noticeable heat capacity effect on temperature is manifested only at the second point after the second heater was enabled corresponding to a non-stationary heating of this rock mass section.

Figures 5 and 6 show that the results of thermal stress calculations are most sensitive to the heat release from the heater and are approximately equally (regardless of position and time) sensitive to the mechanical characteristics of the medium, such as linear coefficient of thermal expansion and Young's modulus.

Based on the results obtained, the following conclusions considered essential for further experiment planning can be made:

- power of the heaters contributes to the largest extent to the temperature and thermal stresses evolving within the entire rock volume, thus, heating provided under the experiment, should (taking into account the size of the installation) correspond well to the expected heat release from VHLW;

- under a steady state mode (400 days or more), pronounced effect on bedrock thermal conductivity was 


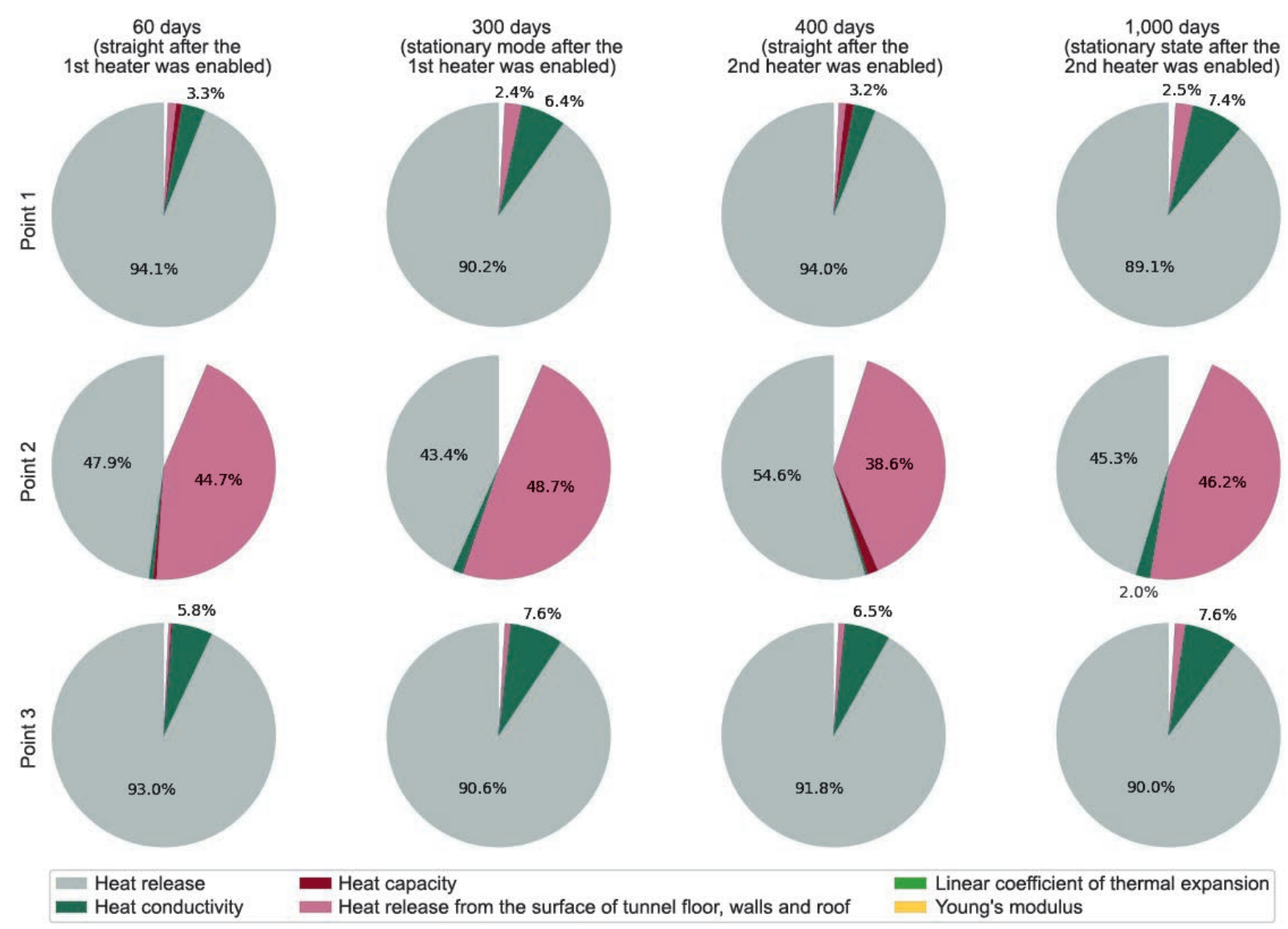

Figure 3. Direct contribution of model parameters to temperature assessment uncertainty
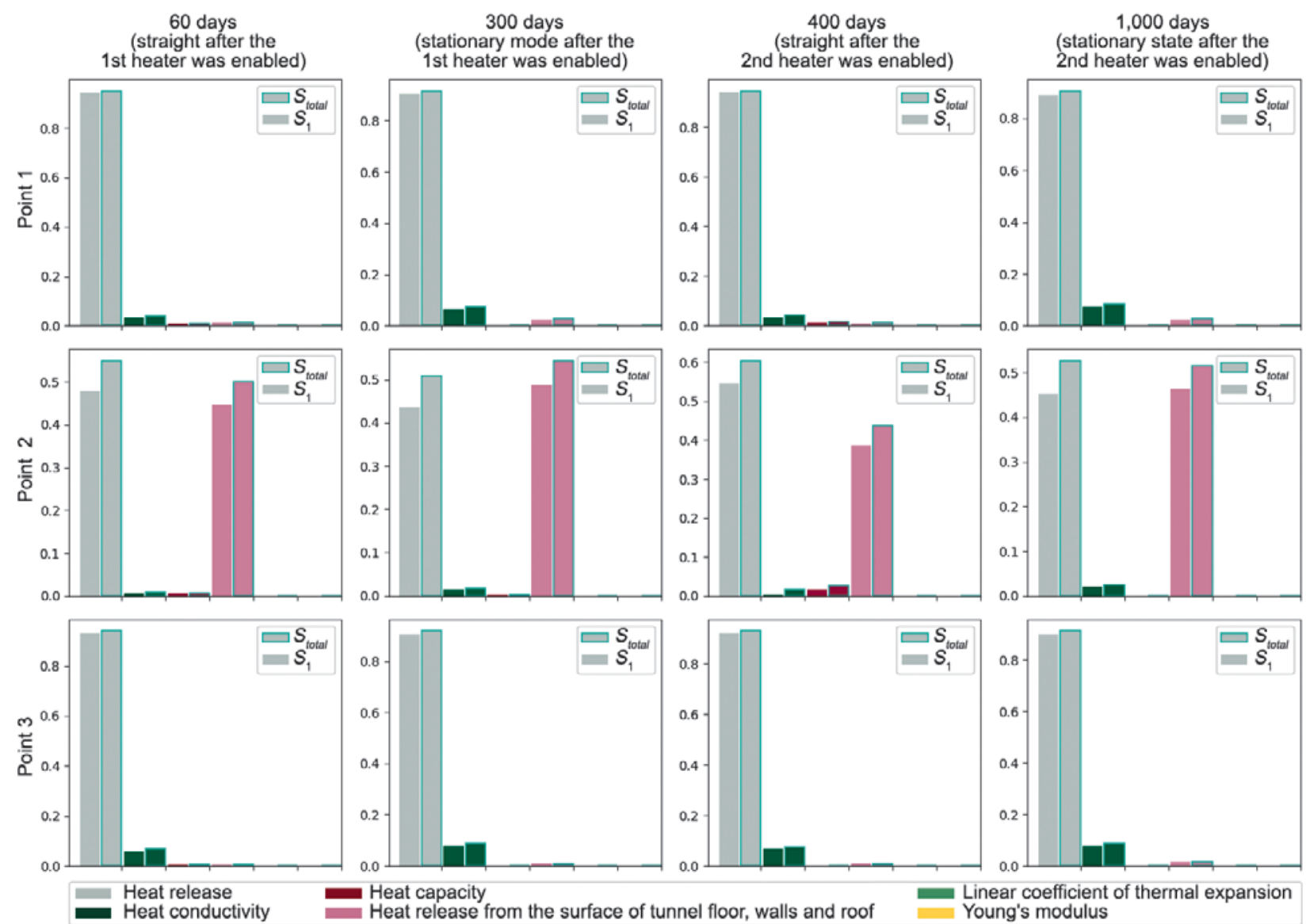

Figure 4. Indices accounting for full and direct contribution of model parameters to the temperature assessment uncertainty 

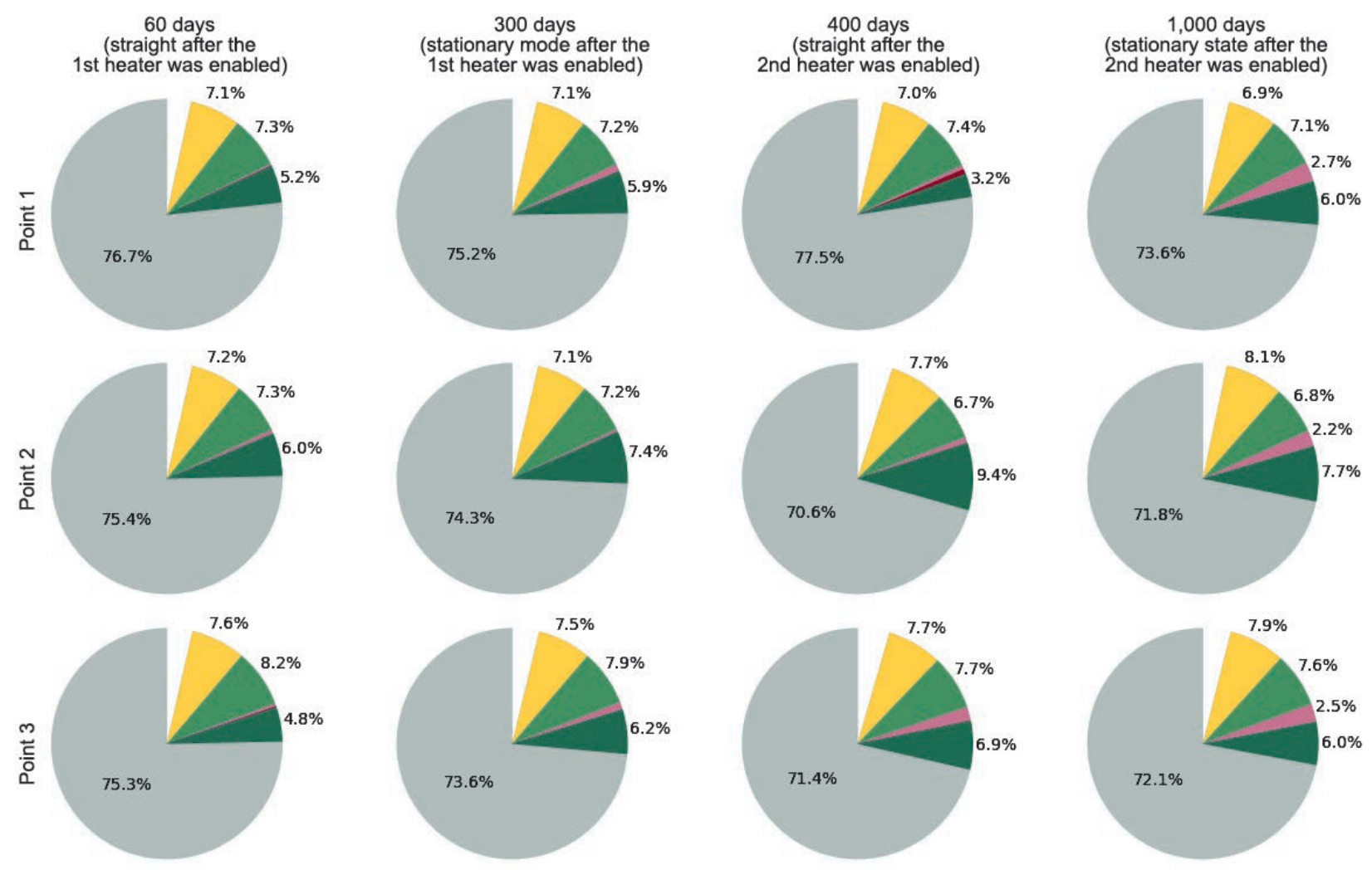

$\begin{array}{lll}\text { Heat release } & \text { Heat capacity } & \text { Linear coefficient of thermal expansion } \\ \text { Heat conductivity } & \text { Heat release from the surface of tunnel floor, walls and roof } & \text { Young's modulus }\end{array}$

Figure 5. Direct contribution of model parameters to the thermal stress assessment uncertainty
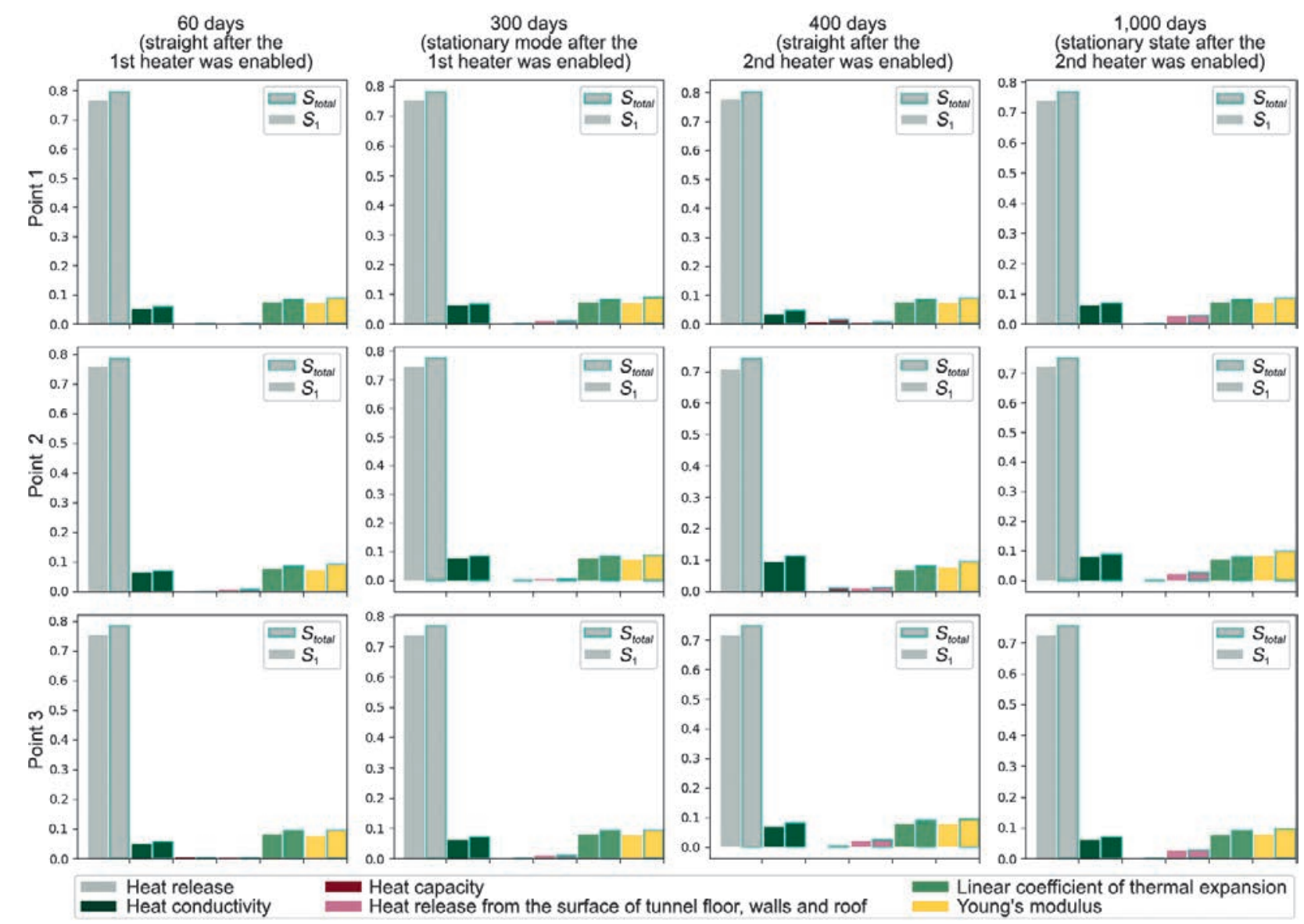

Figure 6. Indices accounting for full and direct contribution of model parameters to the thermal stress uncertainties 
demonstrated, i.e. it was confirmed that the considered experimental designs can be used to refine the data on the thermal conductivity of the host rock;

- no influence of tunnel ventilation was revealed deep within the rock mass, therefore, during the experiment, uncertainties associated with the operation of ventilation system will not affect the estimated thermal conductivity for the main part of the rock mass;

- thermal stresses are influenced by mechanical characteristics of the medium; the influence of thermal conductivity can be noted as well, which also indicates the opportunities for evaluating these characteristics based on the experimental results.

\section{Conclusion}

Sensitivity of a numerical model developed for the thermomechanical experiment was evaluated demonstrating uneven effect of the input parameters depending on the time and spatial distribution in the domain assumed under temperature calculations. For example, thermal conductivity influence on temperature field calculated at the points located at an equal distance from the boreholes appears to be higher under a steady heating mode than at the beginning, whereas the one in the vicinity of a borehole appears to be much lower. Parameter describing heat release from the floor, walls and roof of the tunnel noticeably affects the result in areas being located close to the tunnel floor, however, its effect appears to be quite minor in deeper areas.

Thermal stress model revealed no uneven effects of input parameters depending on the time and spatial distribution.

The results obtained appear to be helpful in elaborating the experimental designs providing more reasoned choice of the sensor locations and heater operating modes.

\section{References}

1. Svitelman V. S., Saveleva E. A., Butov R. A., Linge In. I., Dorofeev A. N., Tikhonovsky V. L. Informacionno-analiticheskaya platforma programmy issledovanij po obosnovaniyu dolgovremennoj bezopasnosti rossijskogo PGZRO [Informational and Software Environment of the Russian Deep Geological Repository Research Program]. Radioaktivnye otkhody-Radioactive Waste, 2018, no. 2 (3), pp. 79-87. 2. Butov R. A., Drobyshevsky N. I., Moiseenko E. V., Tokarev Yu. N. 2017. Finite element code FENIA verification and application for 3D modelling of thermal state of radioactive waste deep geological repository. J. Phys.: Conf. Ser. 891 012174. DOI: 10.1088/1742-6596/891/1/012174.

3. Savelyeva E. A. Koncepciya programmnogo kompleksa dlya ocenki neopredelennosti pri obosnovanii bezopasnosti punktov zakhoroneniya RAO [The concept of a software package for estimating uncertainty in justifying the safety of disposal sites for radioactive waste]. Yadernaya i radiacionnaya bezopasnost' - Nuclear and Radiation Safety, 2016, no. 4(82), pp. 22-35.

4. Belytschko T., Liu W. K., Moran B. Nonlinear Finite Elements for Continua and Structures, John Wiley \& Sons, New York (2000).

5. Singiresu S. Rao. The Finite Element Method in Engineering. Fifth Edition, Butterworth-Heineman, Elsevier, 2011.726 p. ISBN: 1856176614.

6. Sobol I. M. Global sensitivity indices for nonlinear mathematical models and their Monte Carlo estimates. Mathematics and computers in simulation, 2001, vol. 55, no. 1-3, pp. 271-280.

Information about authors

Gorelov Matvei Michailovich, Junior research associate, Nuclear Safety Institute of the Russian Academy of Sciences (52, Bolshaya Tulskaya St., Moscow, 115191, Russia), e-mail: gorelov@ibrae.ac.ru.

Drobyishevskiy Nikolay Ivanovich, $\mathrm{PhD}$, Senior research associate, Nuclear Safety Institute of the Russian Academy of Sciences (52, Bolshaya Tulskaya St., Moscow, 115191, Russia), e-mail: drobyshevsky@inbox.ru.

Moiseenko Evgeniy Viktorovich, PhD, Senior research associate, Nuclear Safety Institute of the Russian Academy of Sciences (52, Bolshaya Tulskaya St., Moscow, 115191, Russia), e-mail: moi@ibrae.ac.ru.

Saveleva Elena Aleksandrovna, PhD, Head of Laboratory, Nuclear Safety Institute of the Russian Academy of Sciences (52, Bolshaya Tulskaya St., Moscow, 115191, Russia), e-mail: esav@ibrae.ac.ru.

Svitelman Valentina Semenovna, PhD, Research associate, Nuclear Safety Institute of the Russian Academy of Sciences (52, Bolshaya Tulskaya St., Moscow, 115191, Russia), e-mail: svitelman@ibrae.ac.ru.

\section{Bibliographic description}

Gorelov M. M., Drobyshevsky N. I., Moiseenko E. V., Saveleva E. A., Svitelman V. S. Sensitivity Analysis for the Numerical Model of the "Rock thermomechanics" Experiment under URF Conditions. Radioactive Waste, 2020, no. 1(10), pp. 92-100. (In Russian). DOI: 10.25283/2587-9707-2020-1-92-100. 